\title{
ON TENSOR PRODUCT GRAPHS
}

\author{
E. SAMPATHKUMAR
}

(Received 4 January 1973; revised 2 January 1974)

Communicated by G. Szekeres

\begin{abstract}
The tensor product $G \oplus H$ of graphs $G$ and $H$ is the graph with point set $V(G) \times V(H)$ where $\left(u_{1}, v_{1}\right)$ adj $\left(u_{2}, v_{2}\right)$ if, and only if, $u_{1}$ adj $u_{2}$ and $v_{1}$ adj $v_{2}$. We obtain a characterization of graphs of the form $G \oplus H$ where $G$ or $H$ is $K_{2}$.
\end{abstract}

\section{Notation and preliminary results}

As usual, let $K_{p}$ denote the complete graph on $p$ points, $C_{n}$ a cycle of length $n$. For a connected graph $G, n G$ is the graph with $n$ components each being isomorphic to $G$.

REMARK. The tensor product $G \oplus H$ is also called conjunction (Harary (1969)), and Kronecker product (Weichsel 1963)).

Weichsel (1963) has proved Theorem 1 and Corollary 1.1.

THEOREM 1. (Weichsel). For connected graphs $G$ and $H$ the product $G \oplus H$ is connected if and only if either $G$ or $H$ contains an odd cycle.

Corollary 1.1. (Weichsel). If $G$ and $H$ are connected graphs with no odd cycles then $G \oplus H$ has exactly two components.

We now prove

COROLlaRY 1.2. For a connected graph $G$ with no odd cycles, $G \oplus K_{2}=2 G$.

Proof. Let $\left\{a_{i}\right\}$ be the point set of $G$ and $K_{2}$ be the line $b_{1} b_{2}$. By Corollary 1.1, $G \oplus K_{2}$ has exactly two components, say $G_{1}$ and $G_{2}$. If for some point $a_{i_{o}}$ in $G,\left(a_{i_{o}}, b_{1}\right) \in G_{1}$ then $\left(a_{i_{o}}, b_{2}\right) \in G_{2}$. For, if there is a path $\left(a_{i_{o}}, b_{1}\right)\left(a_{i_{1}}, b_{2}\right) \ldots$ $\left(a_{i_{k}}, b_{1}\right)\left(a_{i_{n}}, b_{2}\right)$ then $k$ is even and $G$ has the odd cycle $a_{i_{o}} a_{i_{1}} \cdots a_{i_{k}} a_{i_{o}}$, provided the points $a_{i_{r}}, r=0,1, \cdots, k$ are all distinct. Suppose $a_{i_{r}}=a_{i_{s}}$ for some $r$ and $s$, and $r<s$. Let $s$ be the smallest such integer. Clearly, if $r$ is even (odd) then $s$ is odd (even) and $G$ has an odd cycle $a_{i_{n}} a_{i_{r+1}} \cdots a_{i_{s-1}} a_{i_{r}}$. Now, the function $f: G \rightarrow G_{1}$ defined by 


$$
\begin{array}{r}
f\left(a_{i}\right)=\left(a_{i}, b_{1}\right) \text { if }\left(a_{i}, b_{1}\right) \notin G_{1} \\
=\left(a_{i}, b_{2}\right) \text { if }\left(a_{i}, b_{1}\right) \notin G_{1}
\end{array}
$$

is clearly an isomorphism. Similarly, $G \cong G_{2}$.

THEOREM 2. For a graph $G, G=H \otimes K_{2}$ if and only if the following conditions I-IV are true.

I. $G$ has an even number of points and lines.

II. G has no odd cycles.

III. If $G$ is connected, the following should hold.

(a) $G$ has a cycle, say $C_{2 n}: x_{1} x_{2} \cdots x_{2 n} x_{1}$ where $n>1$ is odd.

(b) Let $G_{1}$ be the graph obtained from $G$ by removing all lines of $C_{2 n}$. The components of $G_{1}$ should be of the following two types only.

Type I. Components $E$ such that for $1 \leqq r \leqq n$ the point $x_{r}$ of $C_{2 n}$ belongs to $E$ if and only if the point $x_{n+r}$ belongs to a component $E^{\prime}(\neq E)$ isomorphic to $E$.

Type II. Components $F$ such that for $1 \leqq r \leqq n$ the point $x_{r}$ of $C_{2 n}$ belongs to $F$ if and only if $x_{n+r} \in F$. Further, $F$ should satisfy $I, I I$ and III. That is, $F$ should have an even number of points and lines, and should contain a cycle $C_{2 m}$ where $m>1$ is odd, etc.

IV. Suppose $G$ is disconnected. Then the components of $G$ which are not of the form mentioned in III should be in isomorphic pairs.

Proof. Let $G=H \otimes K_{2}, V(H)=\left\{a_{i}\right\}$ and $K_{2}$ be the line $b_{1} b_{2}$. Clearly condition $I$ is true. We observe that points of $G$ are labeled alternately with the elements of the sets $V_{1}=\left\{a_{i}\right\} \times\left\{b_{1}\right\}$ and $V_{2}=\left\{a_{i}\right\} \times\left\{b_{2}\right\}$. This will not be possible if $G$ has odd cycles, and thus II is true.

Let $G$ be connected. Then by Theorem $1, H$ is connected and contains an odd cycle say, $C_{n}=a_{1} a_{2}, \cdots, a_{n} a_{1}$. This implies that $G$ contains the cycle,

$$
C_{2 n}=\left(a_{1}, b_{1}\right)\left(a_{2}, b_{2}\right)\left(a_{3}, b_{1}\right) \cdots\left(a_{n}, b_{1}\right)\left(a_{1}, b_{2}\right) \cdots\left(a_{n}, b_{2}\right)\left(a_{1}, b_{1}\right) .
$$

Consider the graph $G_{1}$ obtained from $G$ by removing all lines of $C_{2 n}$. In a relabeling of the points of $C_{2 n}$ as $x_{1} x_{2} \cdots x_{2 n} x_{1}$ we find that if $\left(a_{r}, b_{1}\right)$ is labeled as $x_{r}$ then $\left(a_{r}, b_{2}\right)$ is labeled as $x_{n+r}$ (we make use of this fact in our proof without any further reference). Let $E$ be a component of $G_{1}$ and $x_{r}=\left(a_{r}, b_{1}\right)$ be a point of $C_{2 n}$ such that $x_{r} \in E$ and $x_{n+r}=\left(a_{r}, b_{2}\right)$ belongs to a different component $E^{\prime}$ of $G_{1}$. We now show that (i) a point $\left(a_{i}, b_{1}\right)$ (not necessarily on $C_{2 n}$ ) belongs to $E$ if and only if $\left(a_{i}, b_{2}\right)$ belongs to $E^{\prime}$, and (ii) $E \cong E^{\prime}$.

Let $\left(a_{i}, b_{1}\right) \in E$. Then in $E$ there is a path

$$
\left(a_{i}, b_{1}\right)\left(a_{i_{1}}, b_{2}\right)\left(a_{i_{2}}, b_{1}\right) \cdots\left(a_{i_{k}}, b_{2}\right)\left(a_{r}, b_{1}\right) \text {. }
$$


This implies that there is a path

$$
\left(a_{i}, b_{2}\right)\left(a_{i_{1}}, b_{1}\right)\left(a_{i_{2}}, b_{2}\right) \cdots\left(a_{i_{k}}, b_{1}\right)\left(a_{r}, b_{2}\right)
$$

and hence $\left(a_{i}, b_{2}\right) \in E^{\prime}$, since $\left(a_{r}, b_{2}\right) \in E^{\prime}$. Likewise, $\left(a_{j}, b_{1}\right) \in E^{\prime}$ implies $\left(a_{j}, b_{2}\right) \in E$. Clearly, the function $f: E \rightarrow E^{\prime}$ defined by

$$
\begin{array}{ll}
f\left(a_{i}, b_{1}\right)=\left(a_{i}, b_{2}\right) & \text { if } \quad\left(a_{i}, b_{1}\right) \in E \\
f\left(a_{i}, b_{2}\right)=\left(a_{i}, b_{1}\right) & \text { if } \quad\left(a_{i}, b_{2}\right) \in E
\end{array}
$$

is an isomorphism.

Suppose now $F$ is a component of $G_{1}$ and $x_{r}=\left(a_{r}, b_{1}\right)$ is a point of $C_{2 n}$ such that both $x_{r}$ and $x_{n+r}=\left(a_{r}, b_{2}\right)$ belong to $F$. We show that in general, a point $\left(a_{i}, b_{1}\right)$ (not necessarily on $\left.C_{2 n}\right)$ belongs to $F$ if and only if $\left(a_{i}, b_{2}\right) \in F$. Let $\left(a_{i}, b_{1}\right) \in F$. Then there is a path

$$
\left(a_{r}, b_{1}\right)\left(a_{i_{1}}, b_{2}\right) \cdots\left(a_{i_{k}}, b_{2}\right)\left(a_{i}, b_{1}\right) .
$$

This implies that there is a path

$$
\left(a_{r}, b_{2}\right)\left(a_{i_{1}}, b_{1}\right) \cdots\left(a_{i_{k}}, b_{1}\right)\left(a_{i}, b_{2}\right)
$$

and since $\left(a_{r}, b_{2}\right) \in F,\left(a_{i}, b_{2}\right) \in F$. Likewise, $\left(a_{j}, b_{2}\right) \in F$ implies $\left(a_{j}, b_{1}\right) \in F$. It follows now that $F=H_{1} \oplus K_{2}$ where $H_{1}$ is the subgraph of $H$ induced by the points $V\left(H_{1}\right)=\left\{a_{i}:\left(a_{i}, b_{1}\right) \in F\right\}$, and hence $F$ should satisfy I, II and III. This proves that $G_{1}$ can have only two types of components as mentioned in III. If $G$ is disconnected, a component $G_{i}$ of $G$ which is not of the form mentioned in III corresponds to a component $H_{i}$ of $H$ which does not have an odd cycle and so $H_{i} \oplus K_{2}=2 H_{i}=2 G_{i}$ by Corollary 1.2 . Thus $G$ should have such components in pairs and such components are even in number.

Conversely, let the conditions I-IV hold good for a graph $G$ having $2 m$ points. Let $A=\left\{a_{1}, a_{2}, \cdots, a_{m}\right\}$ and $B=\left\{b_{1}, b_{2}\right\}$. We label the points of $G$ with $2 m$ elements of $A \times B$ such that

(i) no two elements of $A \times\left\{b_{1}\right\}$ or $A \times\left\{b_{2}\right\}$ are adjacent, and

(ii) the function $f: G \rightarrow G$ defined by

$$
\begin{aligned}
& f\left(a_{i}, b_{1}\right)=\left(a_{i}, b_{2}\right) \\
& f\left(a_{i}, b_{2}\right)=\left(a_{i}, b_{1}\right)
\end{aligned}
$$

is an isomorphism. The given conditions I-IV ensure that this is indeed possible. Suppose now $G$ is connected. By hypothesis it contains a cycle $C_{2 n}$ where $n$ is odd. We label the points of this cycle successively with the $2 n$ elements

$$
\left(a_{1}, b_{1}\right)\left(a_{2}, b_{2}\right)\left(a_{3}, b_{1}\right) \cdots\left(a_{n}, b_{1}\right)\left(a_{1}, b_{2}\right) \cdots\left(a_{n}, b_{2}\right) .
$$

The above isomorphism takes the cycle into itself and the subgraph $G_{1}$ described 
in III into itself. In case $G_{1}$ is disconnected the isomorphism maps a component of type I into an isomorphic component of the same type, while a component of type II is mapped onto itself. The labeling of the points of $G$ is illustrated in Figure 1, where for convenience we write $i$ for $a_{i}$ and $i^{\prime}$ for $b_{i}$. In this labeling we observe that $\left(a_{i}, b_{1}\right)$ is adjacent to $\left(a_{j}, b_{2}\right)$ if and only if $\left(a_{i}, b_{2}\right)$ is adjacent to $\left(a_{j}, b_{1}\right)$. Now, consider the graph $H$ constructed on the points set $A$ as follows. In $H, a_{i}$ adj $a_{j}$ if and only if $\left(a_{i}, b_{1}\right)$ adj $\left(a_{j}, b_{2}\right)$. It follows now that $G=H \oplus K_{2}$. The graph $G$ in Figure 1 is the tensor product of $H$ and $K_{2}$ in Figure 3. If $G$ is disconnected and if a component $G_{i}$ of $G$ is of the form mentioned in III, then $G_{i}=H_{i} \oplus K_{2}$ for some graph $H_{i}$ as above. If a component $G_{j}$ of $G$ is not of this form then by hypothesis they are in isomorphic pairs and $G_{j} \oplus K_{2}=2 G_{j}$ by Corollary 1.2. This completes the proof of the Theorem.

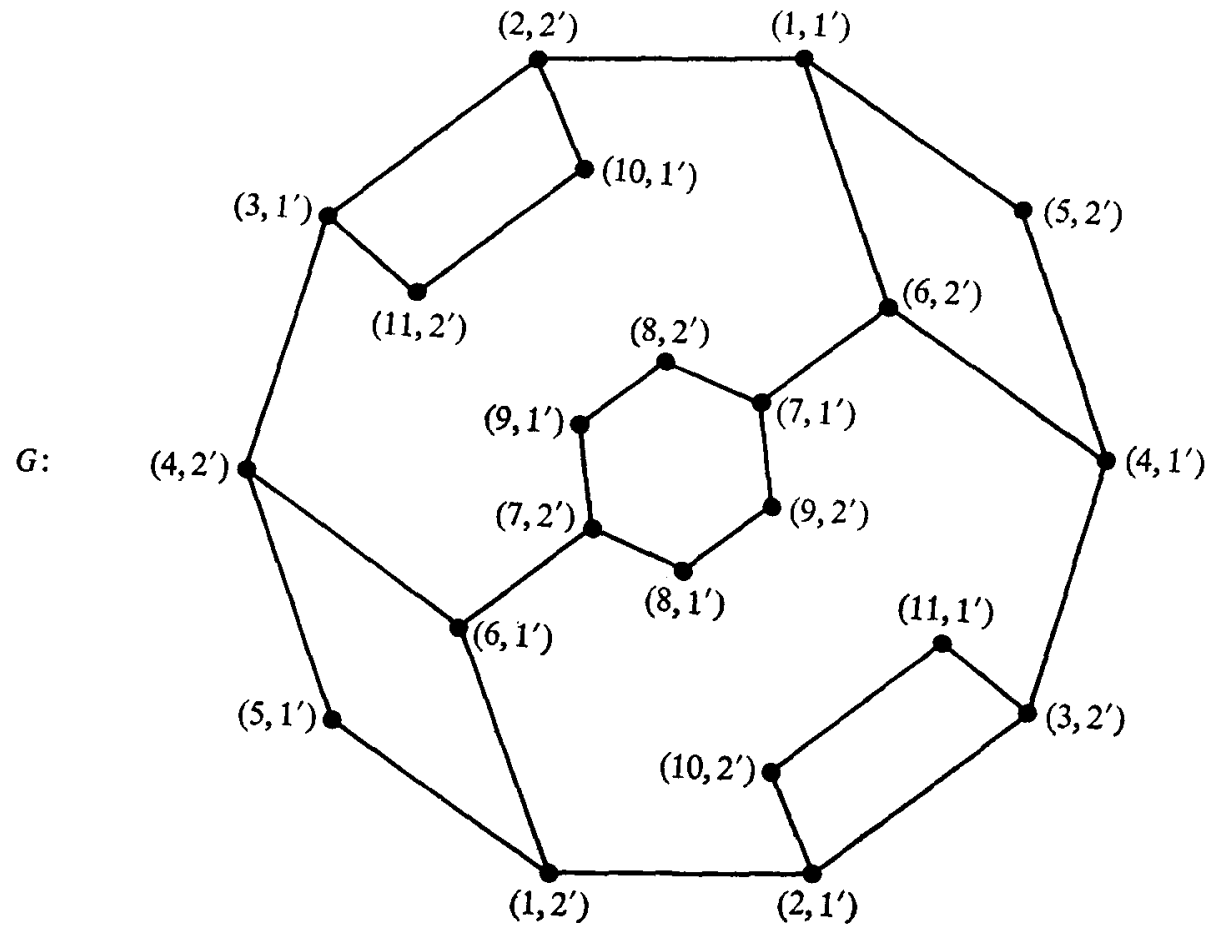

Fig. 1 


$$
\left(5,2^{\prime}\right) \bullet E_{z}
$$

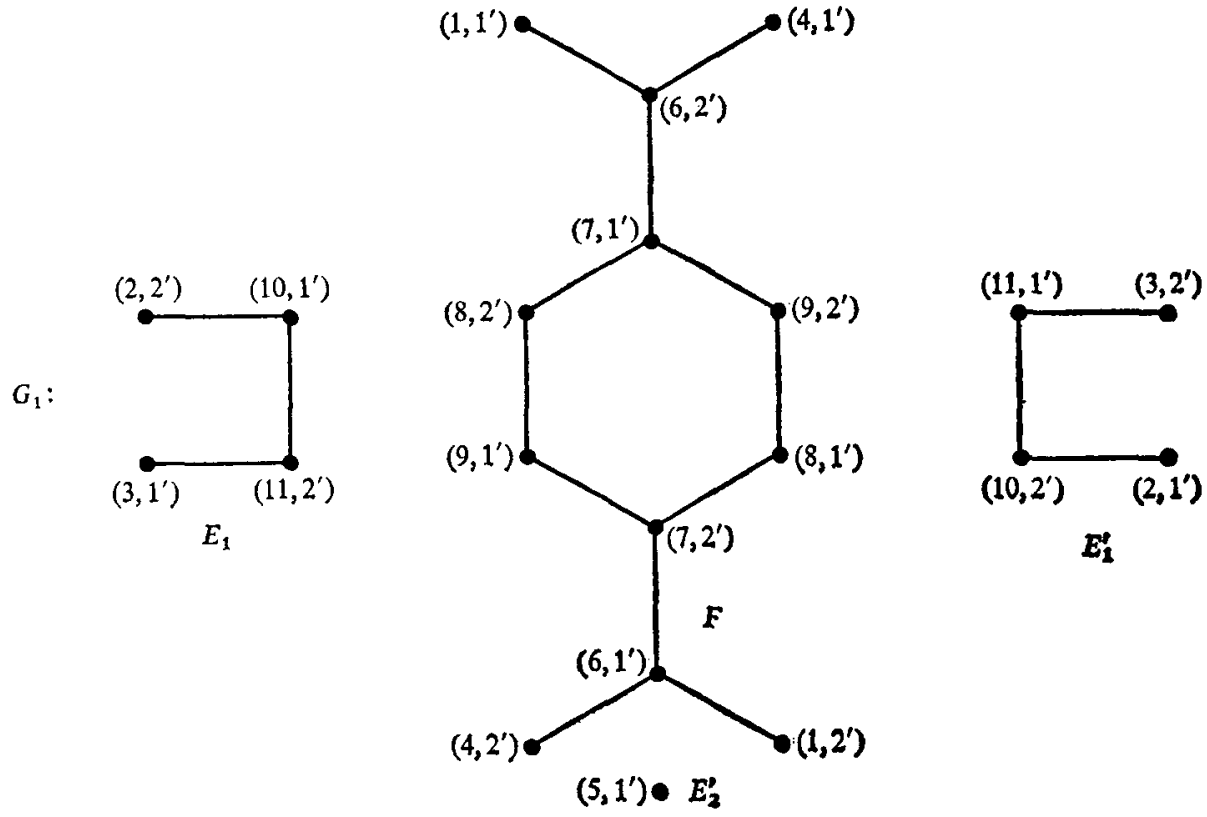

Fig. 2

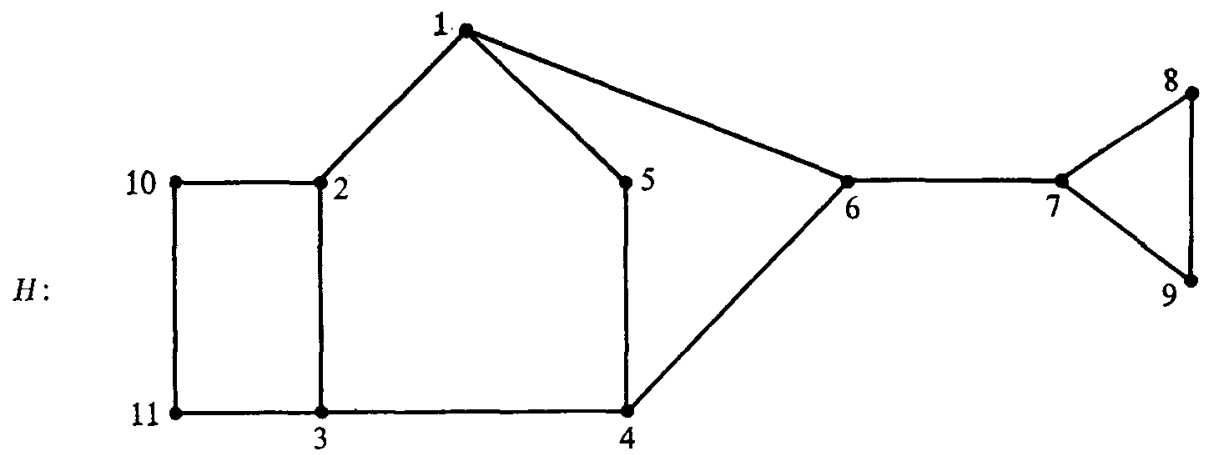

$K: \underset{1^{\prime}}{\longrightarrow}$

Fig. 3

For any two graphs $G_{1}$ and $G_{2}$ we have

$$
G_{1} \oplus G_{2}=G_{1} \oplus \bigcup_{i} e_{i}=\bigcup_{i} G_{1} \oplus e_{i}
$$

where $\left\{e_{i}\right\}$ is the set of lines of $G_{2}$. Each product graph $G_{1} \oplus e_{i}$ is isomorphic to $G_{1} \times K_{2}$. Also, the graphs $G_{1} \oplus e_{i}$ are line disjoint and the number of common 
points (if any) between any two of these graphs is equal to that in $G_{1}$. These observations lead to

THEOREM 3. A necessary condition for a graph $G$ to be the tensor product of two graphs is that $G$ is the line disjoint union of a number of graphs of the form $\mathrm{H} \oplus \mathrm{K}_{2}$ for some graph $H$, and the number of common points (if any) between any two of these graphs must be that in $H$.

For example, the graph $G$ in Figure 4 is the line disjoint union of $K_{1.3} \otimes K_{2}$ and $K_{1.3} \oplus K_{2}$ as illustrated. The number of common points between these two graphs is that in $K_{1,3}$, namely 4 and $G=K_{1,3} \otimes P_{3}$, where $P_{3}$ is a path of length 2 .

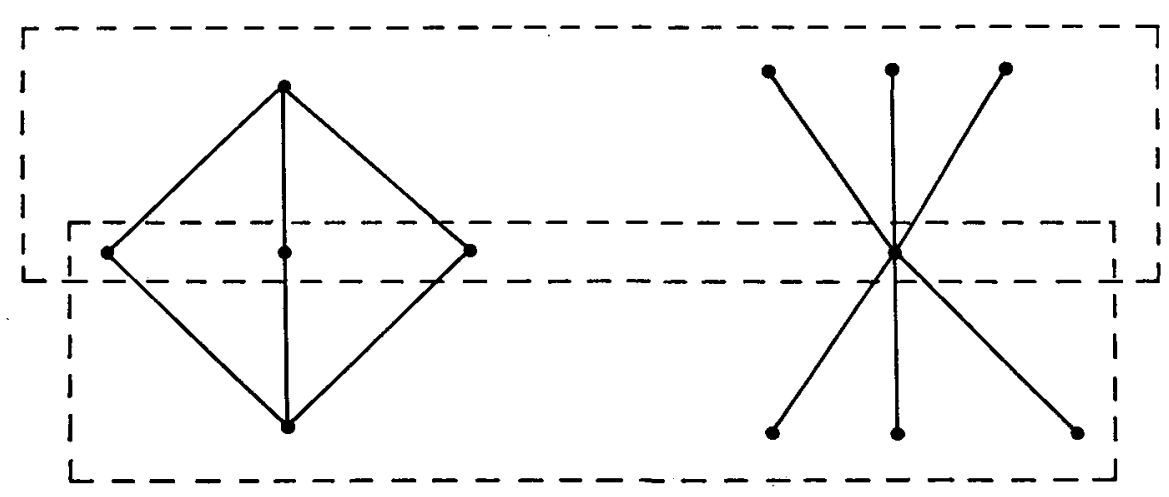

Fig. 4

Thanks are due to the referee for his suggestions.

\section{References}

F. Harary, Graph Theory (Addision-Wesley Publishing Company, Reading Mass. 1969).

P. M. Weichsel (1963), 'The Kronecker product of graphs'. Proc. Amer. Math. Soc. 13, 47-52.

Department of Mathematics

Karnatak University

Dharwar - 58003

Karnatak (India) 